\title{
Enquête sur une éclosion importante de SRAS-CoV-2 dans un établissement de soins de longue durée au début de la pandémie
}

\author{
Michelle Murti MD MSP, Monika Goetz MSc, Andrea Saunders IA MSc, Vidya Sunil DMV MSc, \\ Jennifer L. Guthrie PhD, AliReza Eshaghi PhD, Sandra Zittermann PhD, Sarah Teatero MSc, \\ Nahuel Fittipaldi PhD, Heather Rilkoff MSP, Jonathan B. Gubbay MBBS MMedSc, Gary Garber MD, \\ Sandra Callery IA MScS, Anne Marie Holt MScS, A. Lynn Noseworthy MD MScS
}

Citation : CMAJ 2021 May 10;193:E681-8. doi : 10.1503/cmaj.202485-f

Voir la version anglaise de l'article ici : www.cmaj.ca/lookup/doi/10.1503/cmaj.202485

\section{RÉSUMÉ}

CONTEXTE : Le déploiement de mesures de gestion des éclosions de SRAS-CoV-2 dans les établissements de soins de longue durée en Ontario a permis d'en réduire la fréquence et la gravité. Nous décrivons ici les données épidémiologiques et de laboratoire d'une de ces premières éclosions en Ontario afin de déterminer les facteurs associés à son importance et les impacts des interventions progressives de lutte contre les infections appliquées pendant la durée de l'éclosion.

MÉTHODES : Nous avons obtenu du bureau de santé la liste des cas et les données de l'éclosion afin de décrire les cas chez les résidents et le personnel, leur gravité et leur distribution dans le temps et à l'intérieur de l'établissement touché. Quand elles étaient disponibles, nous avons obtenu des données concernant les échantillons soumis au laboratoire de Santé publique Ontario et effectué un séquençage complet et une analyse phylogénétique des échantillons viraux de l'éclosion.

RÉSULTATS : Sur les 65 résidents de l'établissement de soins de longue durée, 61 (94\%) ont contracté le SRASCoV-2, le taux de létalité étant de $45 \%$ (28/61). Parmi les 67 employés initiaux, $34(51 \%)$ ont contracté le virus, et aucun n'est décédé. Lorsque l'éclosion a été déclarée, 12 employés, 2 visiteurs et 9 résidents présentaient des symptômes. Parmi les résidents, les cas se trouvaient dans 3 des 4 secteurs de l'établissement. L'analyse phylogénétique a montré une forte similitude des séquences; une seule autre souche de SRAS-CoV-2 génétiquement distincte a été identifiée chez un employé à la troisième semaine de l'éclosion. Après le déploiement de toutes les mesures de gestion de l'éclosion, aucun cas n'a été identifié parmi les 26 nouveaux employés appelés en renfort.

INTERPRÉTATION : La propagation rapide et non détectée du virus dans un établissement de soins de longue durée a donné lieu à des taux élevés d'infection chez les résidents et le personnel. L'application progressive de mesures de gestion après le pic de l'éclosion a permis d'éviter la contamination du personnel appelé en renfort et fait désormais partie des politiques à long terme de prévention des éclosions en Ontario.

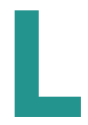

es résidents des établissements de soins de longue durée (ESLD) sont surreprésentés dans la répartition des cas de COVID-19 et de SRAS-CoV-2 au Canada et ailleurs dans le monde $\mathrm{e}^{1-3}$. Les adultes âgés des ESLD sont vulnérables au SRAS-CoV-2 et ils courent un risque accru de complications et de décès en raison de leurs problèmes de santé sous-jacents et de leur âge ${ }^{4}$. En Ontario, les résidents des ESLD représentaient $18 \%$ des cas confirmés pendant la première vague de la pandémie, qui s'est terminée en juin 2020. En date du 6 février 2021, les résidents des ESLD représentaient $5,3 \%$ des cas confirmés de COVID-19, avec un taux de létalité global de 25,4\%, contre 2,4\% pour l'ensemble des cas en Ontario ${ }^{5}$. 
En Ontario, en date du 9 mars 2020, les ESLD avaient reçu des recommandations provinciales sur les mesures optimisées pour prévenir la COVID-19; des directives à jour ont été fournies régulièrement, incluant diverses mesures telles que les indications pour procéder à des tests systématiques, les politiques relatives aux visites, l'interdiction de travailler dans plus d'un ESLD, le port universel du masque et la définition et la gestion strictes des éclosions ${ }^{6}$. Le déploiement de ces mesures ${ }^{7}$ a coïncidé avec une réduction du nombre et de la gravité des éclosions pendant la première vague de la pandémie en Ontario ${ }^{8}$.

Nous présentons ici un rapport sur les données épidémiologiques, les mesures de prévention et de contrôle des infections $(\mathrm{PCl})$ et les analyses de laboratoire et phylogénétiques de l'une des premières et plus importantes éclosions de COVID-19 à survenir dans un ESLD en Ontario, et sur les impacts des mesures progressives de gestion de l'éclosion qui ont guidé les recommandations provinciales subséquentes pour la gestion des éclosions dans les ESLD.

\section{Méthodes}

\section{Sources des données et analyse épidémiologique}

Les éclosions d'infections respiratoires et les cas de COVID19 dans les ESLD doivent obligatoirement être signalés à la santé publique en Ontario en vertu de la Loi sur la protection et la promotion de la santé9. À partir des données extraites du Système intégré d'information sur la santé publique (SIISP) le 11 juin 2020, nous avons analysé les cas de COVID-19 chez le personnel, les résidents et les proches des résidents (visiteurs) qui ont été liés à cette éclosion ${ }^{10}$. Nous avons extrait des données sur l'âge, le sexe, la date d'apparition des symptômes, la nature des symptômes, la date de la collecte des échantillons et l'historique des analyses de laboratoire concernant ces cas.

Nous avons catégorisé les cas confirmés et probables d'après les définitions provinciales à l'époque, selon lesquelles les cas confirmés s'appuyaient sur les résultats des analyses de laboratoire pour le SRAS-CoV-2; les cas probables se définissaient comme suit : 2 symptômes ou plus (température anormale, léthargie, congestion nasale, toux nouvelle ou aggravée, perte d'appétit, enrouement, difficulté à déglutir ou maux de gorge) apparus le 9 mars 2020 ou après, et absence d'analyses de laboratoire ou résultats non concluants $^{11}$. Nous avons obtenu les listes des employés et des résidents présents lors du déclenchement de l'éclosion pour déterminer la population à risque (dénominateur). Aucun nouveau résident n'a été admis dans l'établissement pendant l'éclosion, et du personnel a été appelé en renfort tout au long de l'éclosion. Nous avons obtenu des renseignements sur les mesures de $\mathrm{PCl}$ appliquées en consultant le sommaire de la gestion de l'éclosion produit par le bureau de santé publique. Nous avons ensuite situé géographiquement les résidents selon l'occupation des chambres au début de l'éclosion, puis la progression des cas confirmés et probables par chambre simple, double ou quadruple.

\section{Analyses de laboratoire}

Initialement, les analyses étaient principalement effectuées au laboratoire de Santé publique Ontario; plus tard, les échantillons ont été testés dans tout le réseau des laboratoires ontariens. Le laboratoire de Santé publique Ontario utilisait soit son propre test ciblant le gène de l'enveloppe (E) du SRAS-CoV-2, ou un essai commercial ciblant les gènes E et orflab (test cobas pour le SRAS-CoV-2 de Roche Diagnostics) ${ }^{12}$.

Nous avons procédé au séquençage du génome complet du SRAS-CoV-2 des échantillons qui contenaient suffisamment de virus au laboratoire de Santé publique Ontario (pour plus de renseignements techniques, consulter l'annexe 1, accessible en anglais au www.cmaj.ca/lookup/ doi/10.1503/cmaj.202485/tab-related-content). Les séquences consensus ont été soumises au GISAID (www. gisaid.org/), et les numéros d'accès sont présentés à l'annexe 2, accessible en anglais au www.cmaj.ca/lookup/ doi/10.1503/cmaj.202485/tab-related-content.

\section{Analyse statistique}

Pour le volet épidémiologique, nous avons calculé le taux d'attaque des cas confirmés et probables de COVID-19 parmi les résidents et le personnel, soit le pourcentage de la population à risque ayant contracté le SRAS-CoV-2 pendant l'éclosion.

Pour le volet analytique, nous avons évalué les échantillons positifs selon la valeur de cycle seuil du gène $E$ du test du laboratoire de Santé publique Ontario (indéterminés : 38,1-39,9; négatifs : $\geq 40$ ) et du test cobas de Roche (valeur de cycle seuil correspondant à un résultat négatif non spécifiée par le fabricant). La valeur de cycle seuil représente le nombre de cycles d'amplification en chaîne par polymérase requis pour détecter la cible; elle est inversement proportionnelle à la quantité de virus dans l'échantillon. Nous avons utilisé le test $t$ de Student pour échantillons indépendants avec la version 7.1 du logiciel SAS Enterprise Guide pour comparer les valeurs de cycle seuil moyennes des échantillons initiaux provenant de cas asymptomatiques et symptomatiques et des échantillons recueillis 14 jours ou moins et plus de 14 jours après l'apparition des symptômes (pour les cas symptomatiques).

\section{Approbation éthique}

La Commission de l'évaluation éthique de Santé publique Ontario a déterminé que ce projet ne nécessitait pas l'approbation d'un comité d'éthique de la recherche puisque les activités décrites dans ce manuscrit ont été menées en conformité avec le mandat juridique de Santé publique Ontario, qui est de «fournir des conseils et un soutien scientifiques et techniques à ceux qui œuvrent au sein des divers secteurs pour protéger et améliorer la santé de la population ontarienne " (Loi de 2007 sur l'Agence ontarienne de protection et de promotion de la santé, L.O. 2007, chap. 10), et sont donc considérées comme une initiative de santé publique et non comme de la recherche. 


\section{Résultats}

Un groupe de résidents présentant des symptômes respiratoires aigus a initialement été identifié dans un ESLD le 17 mars 2020; 3 d'entre eux ont reçu un résultat positif au test de dépistage du SRAS-CoV-2 le 20 mars. Les mesures initiales de lutte contre les éclosions ont été mises en place le soir du 17 mars, conformément aux directives préconisées en cas d'éclosion de maladie respiratoire en 2018; l'éclosion a été déclarée le 18 mars, selon la définition provinciale des éclosions d'infections respiratoires, en l'absence de directives et de définitions propres à la COVID19 (figure 1$)^{9,13}$. D'autres mesures propres à la COVID-19 ont été introduites le 20 mars, y compris des précautions contre la transmission par gouttelettes et par contacts et le port universel du masque pour le personnel. Tous les ESLD de l'Ontario avaient interdit les visites en date du 14 mars, par une directive provinciale $\mathrm{e}^{11}$.

Entre le 20 et le 26 mars, des échantillons prélevés chez 28 employés et 2 visiteurs ont été déclarés positifs pour le
SRAS-CoV-2. Lors d'entrevues, 12 employés, 2 visiteurs et 9 résidents ont rapporté que leurs symptômes étaient apparus entre le 9 et le 18 mars. Entre le $1^{\text {er }}$ et le 3 avril, l'établissement a commencé à soumettre à un test de dépistage les résidents récemment décédés et tous les résidents symptomatiques, y compris ceux qui avaient présenté des symptômes depuis le début de l'éclosion, et a ainsi détecté 27 autres résidents positifs pour le SRAS-CoV-2. Le 6 avril, les tests ont débuté chez les résidents asymptomatiques (figure 1). Le dernier résident déclaré positif a subi un prélèvement le 10 avril, avec apparition des symptômes le 30 avril. Le dernier membre du personnel déclaré positif a commencé à ressentir des symptômes le 20 avril. L'éclosion a été déclarée résolue le 14 mai 2020.

Au début de l'éclosion, 65 résidents et 67 employés étaient associés à cet ESLD. En incluant les cas confirmés et probables, le taux d'attaque a été de $94 \%$ chez les résidents $(n=61)$ et de $51 \%$ chez le personnel $(n=34)$. La liste des résidents et des employés touchés apparaît au tableau 1. On n'a rapporté aucun cas asymptomatique parmi les employés;

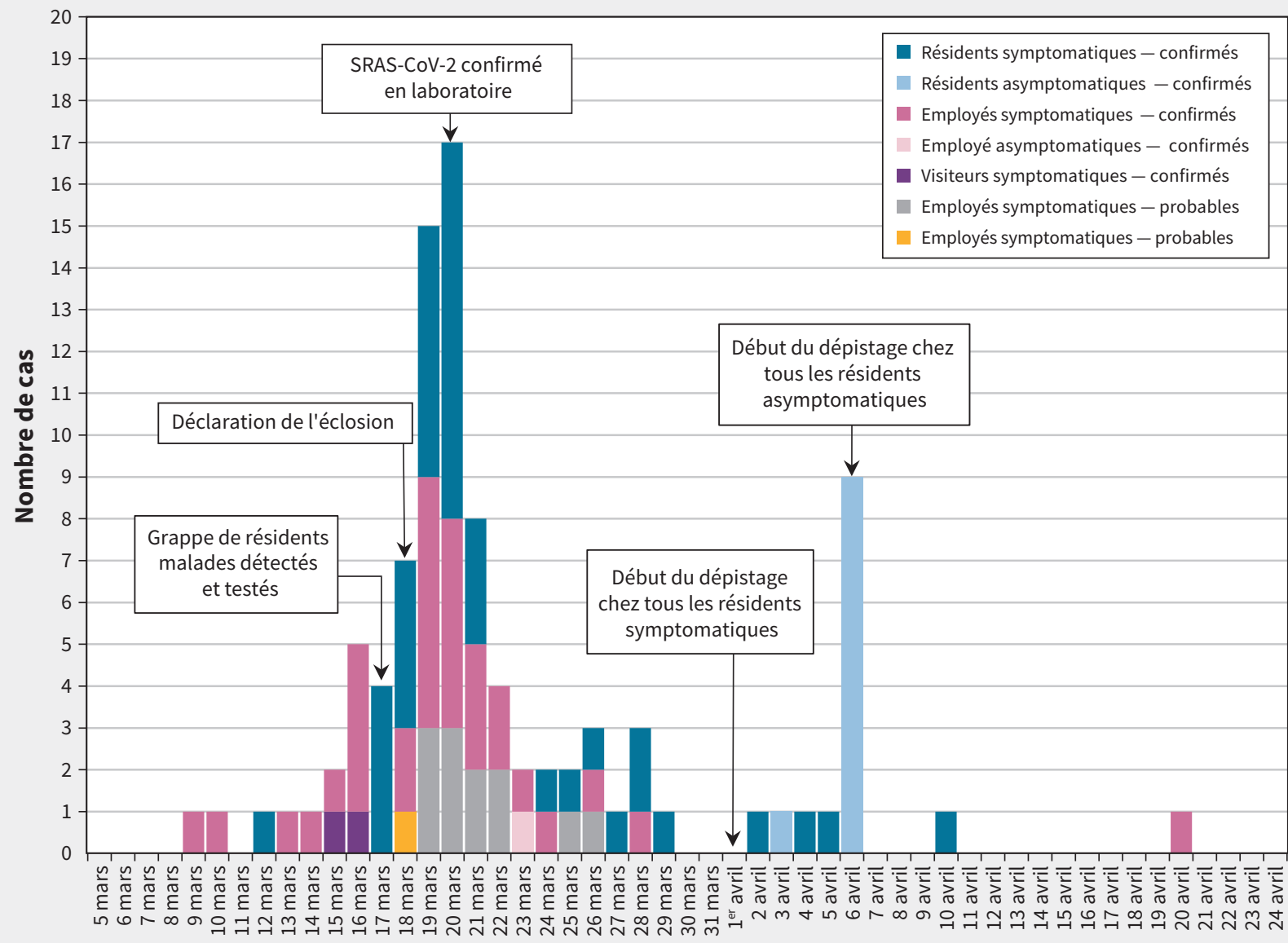

Date d'apparition des symptômes ou de collecte d'échantillions

Figure 1 : Nombre de cas confirmés et probables de COVID-19 chez les résidents, employés et visiteurs ( $n=96)$ associés à une éclosion dans un ESLD, selon la date d'apparition des symptômes ou de collecte des échantillons (si asymptomatiques) en mars et avril 2020. La figure exclut 1 cas survenu chez un résident qui n'a présenté aucun symptôme avant son décès et n'a pas subi de test, mais qui a été considéré comme un cas probable par le bureau de santé. 
Tableau 1 : Caractéristiques démographiques, symptômes et gravité de l'issue des cas confirmés et probables de CoVID-19 chez les résidents $(n=61)$ et employés $(n=34)$ d'un établissement de soins de longue durée en Ontario

\begin{tabular}{|c|c|c|c|c|}
\hline \multirow[b]{2}{*}{ Variable } & \multicolumn{2}{|c|}{$\mathrm{N}^{\text {bre }}(\%)$ de résidents* } & \multicolumn{2}{|c|}{$\mathrm{N}^{\text {bre }}(\%)$ d'employés* } \\
\hline & $\begin{array}{c}\text { Cas confirmés } \\
n=48\end{array}$ & $\begin{array}{c}\text { Cas probables } \\
n=13\end{array}$ & $\begin{array}{c}\text { Cas confirmés } \\
n=33\end{array}$ & $\begin{array}{c}\text { Cas probables } \\
n=1\end{array}$ \\
\hline \multicolumn{5}{|l|}{ Sexe } \\
\hline Hommes & $17(35)$ & $6(46)$ & $1(3)$ & $0(0)$ \\
\hline Femmes & $31(65)$ & $7(54)$ & $32(97)$ & $1(100)$ \\
\hline Âge médian, ans (plage) & $87(45-97)$ & $82(56-98)$ & $51(26-78)$ & 46 \\
\hline \multicolumn{5}{|l|}{ Groupe d'âge, ans } \\
\hline $20-29$ & $0(0)$ & $0(0,0)$ & $2(6)$ & $0(0)$ \\
\hline $30-39$ & $0(0)$ & $0(0,0)$ & $5(15)$ & $0(0)$ \\
\hline $40-49$ & $1(2)$ & $0(0,0)$ & $8(24)$ & $1(100)$ \\
\hline $50-59$ & $3(6)$ & $1(8)$ & $9(27)$ & $0(0)$ \\
\hline $60-69$ & $4(8)$ & $2(15)$ & $8(24)$ & $0(0)$ \\
\hline $70-79$ & $7(15)$ & $3(23)$ & $1(3)$ & $0(0)$ \\
\hline $80-89$ & $18(38)$ & $3(23)$ & $0(0)$ & $0(0)$ \\
\hline$\geq 90$ & $15(31)$ & $4(31)$ & $0(0)$ & $0(0)$ \\
\hline \multicolumn{5}{|l|}{ Ayant présenté des symptômes } \\
\hline Oui & $41 \dagger(85)$ & $12(92)$ & $33 \dagger(100)$ & $1(100)$ \\
\hline Non & $7(15)$ & $1 \ddagger(8)$ & $0(0)$ & $0(0)$ \\
\hline \multicolumn{5}{|l|}{ Principaux symptômes } \\
\hline Toux & $28(68)$ & $8(67)$ & $24(73)$ & $1(100)$ \\
\hline Fièvre ou température anormale & $29(71)$ & $10(83)$ & $20(61)$ & $1(100)$ \\
\hline Léthargie & $17(42)$ & $4(33)$ & $8(24)$ & $0(0)$ \\
\hline Congestion nasale & $14(34)$ & $5(42)$ & $10(30)$ & $0(0)$ \\
\hline Céphalées & $2(5)$ & $0(0)$ & $25(76)$ & $0(0)$ \\
\hline Mal de gorge & $6(15)$ & $0(0)$ & $14(42)$ & $0(0)$ \\
\hline Fatigue & $0(0)$ & $0(0)$ & $18(54)$ & $0(0)$ \\
\hline Anorexie & $7(17)$ & $2(17)$ & $6(18)$ & $0(0)$ \\
\hline Frissons & $1(12)$ & $0(0)$ & $9(27)$ & $1(100)$ \\
\hline Malaise & $0(0)$ & $0(0)$ & $11(33)$ & $0(0)$ \\
\hline \multicolumn{5}{|l|}{ Hospitalisation } \\
\hline Oui & $0(0)$ & $0(0)$ & $0(0)$ & $0(0)$ \\
\hline Non & $48(100)$ & $13(100)$ & $33(100)$ & $1(100)$ \\
\hline \multicolumn{5}{|l|}{ Décès } \\
\hline Oui & $15(31)$ & $13(100)$ & $0(0)$ & $0(0)$ \\
\hline Non & $33(69)$ & $0(0)$ & $33(100)$ & $1(100)$ \\
\hline
\end{tabular}

*Sauf indication contraire.

†Inclut 3 résidents et un employé qui étaient asymptomatiques au moment de la collecte initiale des échantillons, dont les résultats au test étaient positifs et qui ont par la suite développé des symptômes.

†Personne décédée sans subir de test. Le bureau de santé a déterminé qu'il s’agissait d’un cas probable.

chez les résidents, $5 \%(n=3)$ ont présenté des symptômes après l'obtention d'un résultat positif au test de dépistage (présymptomatiques), et $13 \%(n=8)$ sont demeurés asymptomatiques (y compris 7 cas asymptomatiques confirmés et 1 cas asymptomatique probable chez un résident n'ayant présenté aucun symptôme documenté de COVID-19 et dont le décès a été attribué au SRAS-CoV-2 après évaluation par le bureau de santé). La plupart des résidents et employés symptomatiques ont fait état de toux et de fièvre ou d'une température anormale. Chez les employés, les céphalées étaient le symptôme le plus fréquent. Aucun résident $\mathrm{ni}$ employé n'a été hospitalisé en raison de la COVID-19. Par 
Tableau 2 : Propagation du SRAS-CoV-2 chez les résidents $(n=65)$ d'un ESLD selon le nombre de lits par chambre (mars et avril 2020)*

\begin{tabular}{|c|c|c|c|c|c|}
\hline \multirow[b]{2}{*}{$\begin{array}{l}N^{\text {bre }} \text { de lits par } \\
\text { chambre }\end{array}$} & \multicolumn{4}{|c|}{$\begin{array}{l}N^{\text {bre }} \text { de cas probables et confirmés de COVID-19 chez les résidents } \\
\text { ( } \mathbf{N}^{\text {bre }} \text { cumulatif de chambres touchées) }\end{array}$} & \multirow[b]{2}{*}{$\begin{array}{l}\mathrm{N}^{\text {bre }} \text { de résidents } \\
\text { n'ayant jamais } \\
\text { répondu aux critères } \\
\text { de la définition de cas } \\
\qquad n=4\end{array}$} \\
\hline & $\begin{array}{c}12 \text { au } 17 \text { mars, avant } \\
\text { déclaration de } \\
\text { l'éclosion } \\
n=5\end{array}$ & $\begin{array}{c}18 \text { au } 21 \text { mars, pic } \\
\text { de l'éclosion } \\
n=30\end{array}$ & $\begin{array}{l}22 \text { mars au } 5 \text { avril, } \\
\text { avant début du } \\
\text { dépistage } \\
\text { asymptomatique } \\
n=16\end{array}$ & $\begin{array}{c}6 \text { au } 10 \text { avril, après } \\
\text { début du dépistage } \\
\text { asymptomatique } \\
n=10\end{array}$ & \\
\hline Deux & $1(1)$ & $16(12)$ & $6(13)$ & $2(13)$ & 1 \\
\hline Quatre & $3(2)$ & $12(7)$ & $7(8)$ & $7(8)$ & 3 \\
\hline
\end{tabular}

ailleurs, il est important de mentionner que la plupart des résidents avaient une ordonnance de ne pas réanimer $(n=60)$. Le taux de mortalité était de $46 \%(n=28)$ chez les 61 résidents dont l'infection par le SRAS-CoV-2 était confirmée ou probable; aucun décès n'a été signalé parmi les employés. Les 2 visiteurs ont dû être hospitalisés pour la COVID-19 et l'un est décédé.

Les résidents dont les symptômes sont apparus avant la déclaration de l'éclosion vivaient dans 3 des 4 secteurs de l'établissement et occupaient des chambres simples, doubles et quadruples (tableau 2). Le cas index chez les résidents occupait une chambre quadruple. En date du 21 mars, après le pic de l'éclosion chez les résidents, tous les secteurs de l'établissement étaient touchés.

Au début de l'éclosion, on a connu une grave pénurie de personnel en raison du taux d'attaque élevé et rapide chez les employés. Du personnel a été appelé en renfort $(n=26)$ au cours de l'éclosion. À notre connaissance, tous les cas parmi les employés sont survenus chez les 67 membres du personnel originaux, et non chez les nouveaux venus, pour un taux d'attaque final de $37 \%$ chez les employés (34 cas sur 93 employés). Avant la déclaration de l'éclosion (18 mars), les employés ont continué à travailler même s'ils avaient des symptômes en raison de la pénurie de personnel préexistante. Certains employés ont reçu l'autorisation de travailler masqués après l'amélioration de leurs symptômes; ils ont été affectés aux résidents qui avaient reçu un résultat positif au test de dépistage du SRAS-CoV-2, mais ont continué de se confiner à la maison hors des heures de travail. Même si des mesures de $\mathrm{PCl}$ propres à la COVID-19 ont été appliquées à partir du 20 mars (y compris prévention de la contamination par gouttelettes et par contacts chez tous les résidents, symptomatiques ou non), le manque flagrant d'équipement de protection individuelle tôt au cours de l'éclosion a nui à son utilisation ou mené à sa réutilisation par le personnel.

Nous avons obtenu les valeurs de cycle seuil pour 79 échantillons initiaux et 31 échantillons de suivi. Parmi les 79 échantillons initiaux, 69 provenaient de cas symptomatiques et 10 de cas asymptomatiques. Les valeurs de cycle seuil moyennes pour le gène $E$ étaient significativement plus basses (charge virale plus élevée) dans les échantillons initiaux fournis par des patients symptomatiques (24,7; intervalle de confiance [IC] à $95 \% 22,9-26,5)$ comparativement aux cas asymptomatiques (33,3; IC à $95 \% 31,0-35,6 ; p<0,001)$. Les valeurs de cycle seuil étaient également significativement moindres pour les 60 échantillons fournis par des patients symptomatiques dans les 14 jours suivant l'apparition des symptômes (23,6; IC à $95 \%$ 21,8-25,5), comparativement aux 33 échantillons recueillis plus de 14 jours après l'apparition des symptômes (35,4; IC à $95 \% 34,4-36,4 ; p<0,001)$.

Les échantillons de 24 employés et de 4 résidents étaient disponibles et présentaient des quantités virales suffisantes pour le séquençage du génome. La couverture génomique moyenne était de 99,6\%. Dans l'ensemble, les échantillons liés à l'éclosion présentaient de 6 à 9 polymorphismes mononucléotidiques (ou SNP, pour single-nucleotide polymorphism) par rapport à la souche de référence Wuhan-Hu-1, et tous appartenaient à la lignée B.1. L'analyse phylogénétique a montré que tous les échantillons de SRAS-CoV-2 séquencés étaient génétiquement très similaires; il y avait seulement 0 à 4 SNP de différence entre 2 séquences, quelles qu'elles soient (figure 2 ), et $60,7 \%(n=17)$ formaient un seul groupe sans différences de SNP.

Les données épidémiologiques ont montré qu'un employé (ON-PHL-20-00235 dans la figure 2) était récemment revenu d'un voyage à l'étranger avant la détection de l'éclosion, ce qui pourrait signifier que le virus a été introduit dans l'ESLD par cette personne, car à l'époque, la plupart des cas de la province étaient reliés à des voyages internationaux. Cela dit, la personne est retournée au travail alors qu'il y avait déjà des cas symptomatiques dans l'établissement et avant l'apparition de ses propres symptômes; en outre, la séquence du génome du SRAS-CoV-2 isolé chez cette personne était identique à celle des cas liés à l'éclosion dont les symptômes étaient apparus plus tôt. Il est donc plus probable qu'elle ait été infectée localement, plutôt qu'à l'étranger. En revanche, la séquence de l'échantillon ON-PHL-20-00238, recueilli chez un employé la troisième semaine de l'éclosion (selon la date d'apparition 


\begin{tabular}{|l|}
\hline Type de cas \\
O Employé \\
Résident \\
Apparition des symptômes \\
Semaine 1, du 9 au 15 mars \\
Semaine 2, du 16 au 22 mars \\
Semaine 3, du 23 au 29 mars \\
Semaine 4, du 30 mars au 5 avril \\
\hline
\end{tabular}

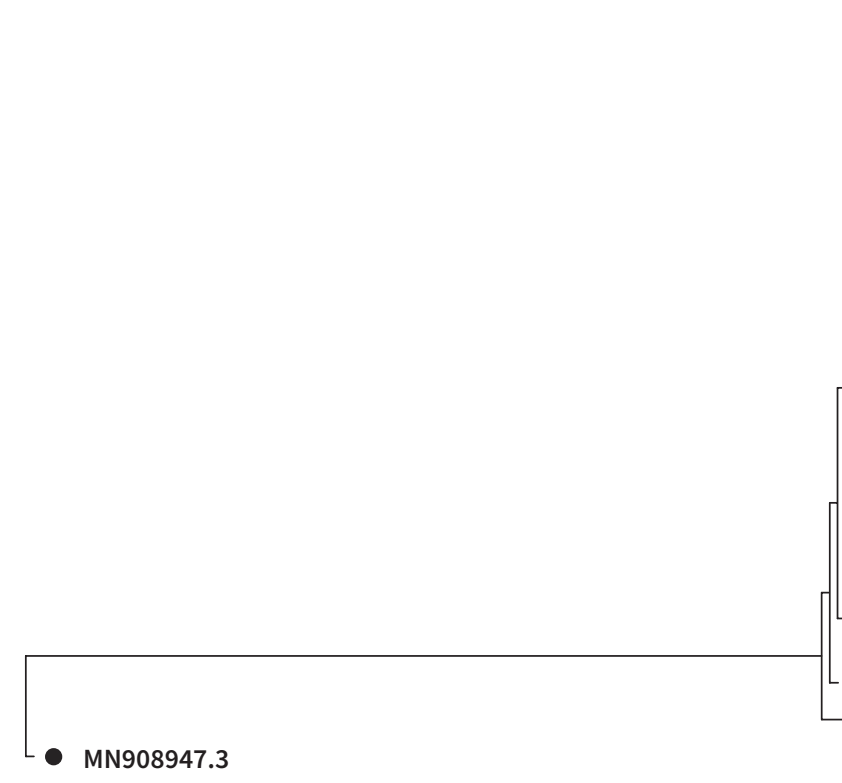

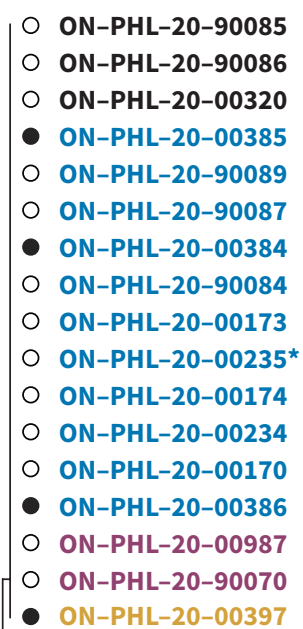

ON-PHL-20-00397

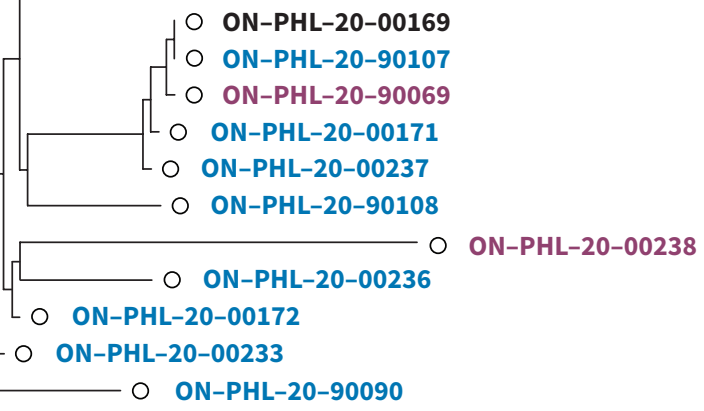

Figure 2 : Arbre de probabilité maximale montrant les liens phylogénétiques entre 28 génomes de SRAS-CoV-2 provenant d'échantillons recueillis chez des résidents et des employés en mars et avril 2020. L'arbre part du génome de référence, Wuhan-Hu-1 (référence : MN908947.3). Remarque : L'analyse phylogénétique illustre les relations évolutionnaires entre les séquences. La longueur des branches horizontales correspond à la distance génétique (nombre de substitutions de nucléotides par site). ON-PHL-20-00238 est génétiquement distinct, avec 3 polymorphismes mononucléotidiques par rapport à la séquence la plus proche. *Un employé avait fait un voyage international avant l'éclosion.

des premiers symptômes), semble génétiquement distincte, avec 3 SNP par rapport à la séquence la plus proche. Étant donné que cette différence est supérieure au nombre attendu de mutations pour le SRAS-CoV-2, cela représente probablement une introduction distincte du virus dans l'établissement ${ }^{14}$. Il a été impossible d'obtenir un échantillon du dernier cas survenu chez les employés pour analyse.

\section{Interprétation}

Les éclosions de SRAS-CoV-2 dans les ESLD durant la pandémie ont été particulièrement importantes ${ }^{15}$. Nous faisons état de l'une des premières éclosions dans un ESLD en Ontario, qui est associée à l'un des plus importants taux d'attaque observés à ce jour, à un moment où il y avait peu de cas dans la région et 274 cas dans la province au total ${ }^{1,16}$. La courbe épidémique, la distribution des cas dans l'établissement, l'analyse phylogénétique et l'évaluation des mesures de $\mathrm{PCl}$ semblent indiquer que l'introduction du virus, survenue avant le déploiement de mesures spécifiques pour la prévention de la COVID-19 dans les ESLD à l'échelle provinciale, s'est rapidement propagée parmi les employés, les résidents et les visiteurs. Toutefois, l'application subséquente de mesures de contrôle a probablement limité la transmission au personnel, car aucun cas n'a été observé parmi les 26 nouveaux employés appelés en renfort durant l'éclosion. Les leçons de cette expérience initiale appuient la validité des mesures de gestion des éclosions actuellement recommandées et la prise en charge rapide et énergique des cas et des éclosions dans les ESLD.

En Ontario, les recommandations initiales de dépistage actif de la COVID-19 chez tous les employés, les résidents et les visiteurs des ESLD n'incluaient pas les symptômes atypiques, fréquents chez les adultes âgés, ni la perte du sens de l'odorat ou du goût, un symptôme fréquent reconnu comme tel plus tard au cours de la pandémie ${ }^{11}$. Les symptômes apparus chez 9 employés avant la déclaration de 
l'éclosion pourraient être passés inaperçus en raison d'une méconnaissance du spectre complet des symptômes de la COVID-19 à l'époque. De plus, la pénurie de personnel préexistante, exacerbée par l'éclosion, pourrait avoir contribué au présentéisme des employés symptomatiques et à la propagation du virus. Le port universel du masque par le personnel n'a pas été imposé avant le 20 mars. Les mesures provinciales stipulent à présent que les employés doivent s'absenter du travail s'ils présentent des symptômes de COVID-19 et qu'ils doivent porter un masque pour exercer un contrôle à la source ${ }^{7}$. Le manque d'effectifs qui perdure dans les ESLD continue de complexifier la gestion des éclosions ${ }^{17}$.

Le nombre de cas chez le personnel et les résidents de l'ESLD avant la déclaration de l'éclosion et le lien entre leurs séquences génomiques semblent indiquer une seule introduction initiale du SRAS-CoV-2 ou l'introduction concomitante de plusieurs personnes infectées par des souches étroitement apparentées, qui a donné lieu à une propagation rapide. La distribution des cas parmi les résidents dans tout l'établissement au début de l'éclosion suggère que le personnel aurait propagé l'infection dans la population de résidents ou qu'une transmission entre résidents est survenue dans les aires communes. La propagation entre résidents dans les chambres à occupation double et quadruple ou dans les aires communes et l'infrastructure globale de l'établissement peut avoir favorisé un taux d'attaque plus élevé, comme le montrent les analyses de la densité et des infrastructures dans les ESLD ${ }^{18,19}$. L'utilisation de la définition de cas provinciale des éclosions d'infections respiratoires probables (2 cas ou plus d'infection respiratoire aiguë sans confirmation en laboratoire), qui a mené à la déclaration de l'éclosion le 17 mars, pourrait avoir retardé la mise en place de l'intervention, le premier résident ayant présenté des symptômes dès le 12 mars $^{9}$. Les directives provinciales en cas d'éclosion de COVID-19 ont depuis été mises à jour et considèrent désormais qu'un seul cas chez les résidents ou les employés est un indicateur d'éclosion dans un ESLD ${ }^{20}$.

Le dépistage initial de tous les résidents et employés dès la déclaration de l'éclosion de SRAS-CoV-2 dans I'ESLD, comme le prévoit désormais la directive provinciale, aurait pu permettre une détection plus rapide et complète des cas de cette éclosion ${ }^{11}$. On recommande aussi actuellement de tester à répétition durant l'éclosion et d'effectuer des tests post mortem pour détecter les cas, ce qui n'était pas le cas au début de l'éclosion. La détection rapide des cas asymptomatiques et présymptomatiques aide à suivre la propagation de l'éclosion et à effectuer un regroupement en cohortes. Un rapport publié sur le dépistage systématique effectué chez des résidents d'établissements de soins de longue durée aux États-Unis a permis de détecter $39,6 \%$ de cas asymptomatiques, en plus des cas symptomatiques ${ }^{21}$. Une étude du Michigan a conclu qu'un dépistage effectué tous les 3-7 jours pendant la durée d'une éclosion dans un établissement de soins de longue durée pourrait être nécessaire pour détecter les cas asymptomatiques ${ }^{22}$. Notre analyse des valeurs de cycle seuil des échantillons recueillis plus de 14 jours après le déclenchement des symptômes et des échantillons provenant de cas asymptomatiques semble indiquer que le dépistage hâtif des cas symptomatiques et le dépistage à répétition chez les personnes asymptomatiques permettraient de réduire le nombre de résultats faussement négatifs en raison de tests non administrés ou administrés trop tard. Les tests sérologiques n'étaient pas disponibles pour évaluer la possibilité de résultats faussement négatifs chez les résidents demeurés asymptomatiques et ayant obtenu des résultats négatifs plus tard au cours de l'éclosion, qui auraient pu avoir été infectés au début de l'éclosion et avoir été rétablis au moment des tests. C'est la seule hypothèse qui expliquerait pourquoi 4 résidents (6\%) ont toujours obtenu des résultats négatifs aux tests de SRAS-CoV-2, malgré leur exposition et leur sensibilité.

Les forces de notre étude incluent une collaboration étroite entre le bureau de santé et l'ESLD, qui a permis une analyse beaucoup plus approfondie des premiers jours de l'éclosion et des difficultés présentes au début de la pandémie au Canada, comparativement à d'autres études de cas. Notre analyse détaillée fournit des pistes additionnelles pour déterminer les facteurs susceptibles d'avoir contribué à la propagation dans l'ESLD. Notre étude montre aussi l'efficacité des mesures de prévention des éclosions déployées pour prévenir l'infection chez les employés: aucune infection subséquente n'a été détectée parmi les employés appelés en renfort après l'application des mesures de gestion de l'éclosion, même si des cas infectieux étaient encore présents. Notre analyse des valeurs de cycle seuil fournit aussi de nouvelles pistes expliquant pourquoi, au cours de la première vague de la pandémie, des cas sont probablement passés inaperçus lorsque des tests ont été effectués tardivement chez des personnes symptomatiques et qu'aucun test de routine n'a été fait chez les personnes asymptomatiques. Le séquençage à grande échelle du génome entier du SRAS-CoV-2 a permis de caractériser l'apparition et la propagation de lignées distinctes, et de déterminer si chaque cas était dû à une infection liée à un voyage, à une transmission locale ou à la présence de foyers d'éclosion multiples, comme nous l'avons observé dans cette analyse $23-25$. Peu d'études sur des éclosions ont inclus une analyse phylogénétique avec leur analyse épidémiologique, comme nous l'avons fait; cette information additionnelle donne d'autres pistes d'explication de la transmission dans l'ESLD, particulièrement au début de la pandémie, alors qu'il y avait généralement peu de cas en Ontario.

\section{Limites de l'étude}

Une des limites de notre analyse génomique est le fait qu'une proportion relativement faible $(<30 \%)$ des échantillons a été séquencée, puisque certains échantillons viraux n'étaient plus disponibles ou présentaient une charge virale insuffisante ne permettant pas une couverture adéquate du génome ${ }^{26,27}$.

Les autres limites de l'étude incluent le fait que les données concernant les visiteurs étaient incomplètes, rendant impossible l'établissement d'un lien avec d'autres cas chez 
les visiteurs, et des données limitées sur les interactions entre employés, entre employés et visiteurs, entre visiteurs et résidents ou entre les résidents et d'autres personnes à l'extérieur de l'ESLD; ces interactions pourraient toutes avoir contribué à une introduction initiale du virus. De plus, les cas chez le personnel et les visiteurs pourraient avoir été sousestimés, si ces personnes n'ont pas subi de tests ou si les tests ont été effectués plus tard au cours de l'éclosion.

\section{Conclusion}

Les leçons apprises de cette première éclosion importante de SRAS-CoV-2 dans un ESLD ont permis de guider les mesures de gestion des éclosions provinciales actuelles, particulièrement l'importance de la détection rapide des symptômes, des cas et des éclosions, et de l'application de mesures additionnelles (dépistage et mesures de prévention). Le dépistage actif et le port universel du masque chez le personnel sont nécessaires pour prévenir l'introduction et la propagation silencieuse du SRAS-CoV-2 par le personnel. Le dépistage systématique chez les résidents et le personnel durant une éclosion permet de détecter rapidement les cas et facilite la mise en place de mesures de lutte contre les éclosions. II faut s'assurer d'avoir du personnel en nombre suffisant et l'équipement de protection individuelle requis pour administrer des soins de façon appropriée, protéger le personnel et prévenir la transmission dans les établissements. Bien que ces mesures de lutte contre la transmission soient efficaces une fois une éclosion déclarée, il ne faut pas oublier que la prévention des éclosions dans les ESLD passe par un faible taux de transmission communautaire et une amélioration de l'infrastructure des ESLD, de la densité des populations qui y résident et de leur dotation en personnel.

\section{Références}

1. COVID-19 data and surveillance. Toronto: Ontario Agency for Health Protection and Promotion (Public Health Ontario); 2020. Accessible ici : https://www. publichealthontario.ca/en/data-and-analysis/infectious-disease/covid-19-data -surveillance (consulté le 4 juill. 2020).

2. Coronavirus disease 2019 (COVID-19): epidemiology update. Ottawa: Government of Canada; 2020. Accessible ici : https://health-infobase.canada.ca/covid -19/epidemiological-summary-covid-19-cases.html (consulté le 4 juill. 2020).

3. Coronavirus disease (COVID-19) weekly epidemiological update and weekly operational update. Geneva: World Health Organization; 2020. Accessible ici : https://www.who.int/emergencies/diseases/novel-coronavirus-2019/situation -reports/ (consulté le 4 juill. 2020).

4. Volling C, Popalyar A, Defalco K, et al.; Public Health Agency of Canada; National Advisory Committee on Infection Prevention and Control (NAC-IPC). Infection prevention and control for COVID-19: interim guidance for long term care homes. Ottawa: Government of Canada; 2020. Accessible ici : https:// www.canada.ca/en/public-health/services/diseases/2019-novel-coronavirus -infection/prevent-control-covid-19-long-term-care-homes.html\#a18 (consulté le 4 juill. 2020).

5. Weekly epidemiologic summary: Focus on January 31, 2021 - February 6, 2021. Toronto: Ontario Agency for Health Protection and Promotion (Public Health Ontario); 2021. Accessible ici : https://www.publichealthontario.ca/-/media/ documents/ncov/epi/covid-19-weekly-epi-summary-report.pdf?la=en (consulté le 13 févr. 2021).

6. Pollard B. COVID-19 updates [memorandum to: long-term care homes sector]. Toronto: Ministry of Long-Term Care; 2020 Mar. 9. Accessible ici : https://www. oha.com/Bulletins/MLTC\%20ADM\%20Memo-COVID19\%20Updates\%20March\% 2009\%202020.pdf (consulté le 4 juill. 2020).
7. COVID-19: orders, directives, memorandums and other resources. Toronto: Ontario Ministry of Health and Long-Term Care; 2020. Accessible ici : http:// www.health.gov.on.ca/en/pro/programs/publichealth/coronavirus/dir_mem_ res.aspx (consulté le 4 juill. 2020).

8. Enhanced epidemiologic summary: COVID-19 in long-term care home residents in Ontario - January 15, 2020 to June 1, 2020. Toronto: Ontario Agency for Health Protection and Promotion (Public Health Ontario); 2021. Accessible ici : https://www.publichealthontario.ca/-/media/documents/ncov/ epi/2020/06/covid-19-epi-ltch-residents.pdf?la=en (consulté le 4 juill. 2020).

9. Appendix A: disease-specific chapters: respiratory infection outbreaks in institutions and public hospitals. Toronto: Ministry of Health and Long-Term Care; 2019. Accessible ici : http://www.health.gov.on.ca/en/pro/programs/ publichealth/oph_standards/docs/respiratory_outbreaks_chapter.pdf (consulté le 4 juill. 2020).

10. Ontario COVID-19 data tool. Toronto: Ontario Agency for Health Protection and Promotion (Public Health Ontario); 2020. Accessible ici : https://www. publichealthontario.ca/en/data-and-analysis/infectious-disease/covid-19 -data-surveillance/covid-19-data-tool (consulté le 4 juill. 2020).

11. COVID-19: guidance for the health sector. Toronto: Ontario Ministry of Health and Long-Term Care; 2020. Accessible ici : http://www.health.gov.on.ca/en/ pro/programs/publichealth/coronavirus/2019_guidance.aspx\#case (consulté le 22 juill. 2020).

12. Corman VM, Kaiser M, Molenkamp R, et al. Detection of 2019 novel coronavirus (2019-nCoV) by real-time RT-PCR. Euro Surveill 2020;25:2000045. doi: 10.2807/1560-7917.ES.2020.25.3.2000045.

13. Control of respiratory infection outbreaks in long-term care homes, 2018. Toronto: Ontario Ministry of Health and Long-Term Care; 2018. Accessible ici : http://www.health.gov.on.ca/en/pro/programs/publichealth/oph_standards/ docs/reference/RESP_Infectn_ctrl_guide_LTC_2018_en.pdf (consulté le 22 juill. 2020).

14. Rambaut A. Phylogenetic analysis of nCoV-2019 genomes. virological.org 2020 Mar. 06. Accessible ici : https://virological.org/t/phylodynamic-analysis -176-genomes-6-mar-2020/356 (consulté le 24 août 2020).

15. Fisman DN, Bogoch I, Lapointe-Shaw L, et al. Risk factors associated with mortality among residents with coronavirus disease 2019 (COVID-19) in long-term care facilities in Ontario, Canada. JAMA Netw Open 2020;3:e2015957. doi: 10.1001/jamanetworkopen.2020.15957.

16. Salcher-Konrad M, Jhass A, Naci H, et al. COVID-19 related mortality and spread of disease in long-term care: a living systematic review of emerging evidence. medRxiv 2020 Aug. 1. Accessible ici : https://doi.org/10.1101/2020.06.09.20125237 (consulté le 4 juill. 2020).

17. Liu M, Maxwell CJ, Armstrong P, et al. COVID-19 in long-term care homes in Ontario and British Columbia. CMAJ 2020;192:E1540-6.

18. Brown KA, Jones A, Daneman N, et al. Association between nursing home crowding and COVID-19 infection and mortality in Ontario, Canada. JAMA Intern Med 2021;181:229-36. Accessible ici : https://jamanetwork.com/ journals/jamainternalmedicine/fullarticle/2772335 (consulté le 13 févr. 2021).

19. Stall NM, Jones A, Brown KA, et al. For-profit long-term care homes and the risk of COVID-19 outbreaks and resident deaths. CMAJ 2020;192:E946-55.

20. COVID-19 outbreak guidance for long-term care homes (LTCH): version 2 April 15, 2020. Toronto: Ontario Ministry of Health; 2020. Accessible ici : http:// www.health.gov.on.ca/en/pro/programs/publichealth/coronavirus/docs/LTCH _outbreak_guidance.pdf (consulté le 24 juill. 2020).

21. Bigelow B, Tang O, Barshick B, et al. Outcomes of universal COVID-19 testing following detection of incident cases in 11 long-term care facilities. JAMA Intern Med 2021;181:127-9.

22. Sanchez GV, Biedron C, Fink LR, et al. Initial and repeated point prevalence surveys to inform SARS-CoV-2 infection prevention in 26 skilled nursing facilities - Detroit, Michigan, March-May 2020. MMWR Morb Mortal Wkly Rep 2020;69:882-6. doi: 10.15585/mmwr.mm6927e1.

23. Rambaut A, Holmes EC, O'Toole Á, et al. A dynamic nomenclature proposal for SARS-CoV-2 lineages to assist genomic epidemiology. Nat Microbiol 2020;5:1403-7.

24. Eden JS, Rockett R, Carter I, et al. An emergent clade of SARS-CoV-2 linked to returned travellers from Iran. Virus Evol 2020;6:veaa027. doi: 10.1093/ve/veaa027.

25. Geoghegan JL, Ren X, Storey M, et al. Genomic epidemiology reveals transmission patterns and dynamics of SARS-CoV-2 in Aotearoa New Zealand. medRxiv 2020 Aug. 20. Accessible ici : https://doi.org/10.1101/2020.08.05.20168930 (consulté le 23 août 2020).

26. Seemann T, Lane CR, Sherry NL, et al. Tracking the COVID-19 pandemic in Australia using genomics. Nat Commun 2020;11:4376. doi: 10.1038/s41467-020-18314-x.

27. Lemieux J. Introduction and spread of SARS-CoV-2 in the greater Boston area. virological.org 2020 June 4. Accessible ici : https://virological.org/t/introduction -and-spread-of-SARS-cov-2-in-the-greater-boston-area/503 (consulté le 23 août 2020). 


\section{Intérêts concurrents : Aucun déclaré.}

Cet article a été révisé par des pairs.

Affiliations : Santé publique Ontario (Murti, Saunders, Guthrie, Eshaghi, Zittermann, Teatero, Fittipaldi, Rilkoff, Gubbay, Garber, Callery); Université de Toronto (Murti, Fittipaldi, Gubbay, Garber), Toronto, Ont.; Bureau de santé du district d'Haliburton, Kawartha et Pine Ridge (Goetz, Sunil, Holt, Noseworthy), Port Hope, Ont.

Collaborateurs : Michelle Murti a élaboré et conçu l'étude. Monika Goetz, Andrea Saunders, Vidya Sunil, Jennifer Guthrie, AliReza Eshaghi, Sandra Zittermann, Sarah Teatero, Nahuel Fittipaldi, Heather Rilkoff, Jonathan Gubbay, Gary Garber, Sandra Callery, Anne Marie Holt et A. Lynn Noseworthy ont recueilli, analysé et interprété les données. Michelle Murti a rédigé le manuscrit, dont tous les auteurs ont révisé de façon critique le contenu intellectuel. Tous les auteurs ont approuvé la version définitive pour publication et ont accepté de se porter garants de tous les aspects du travail.

Financement : Ces travaux ont été rendus possibles grâce aux mandats organisationnels de Santé publique Ontario et du Bureau de santé du district d'Haliburton, Kawartha et Pine Ridge. La collecte et l'analyse des données génomiques ont en partie été rendues possibles par une subvention de Génome Canada, versée à Nahual Fittipaldi pour le Réseau canadien de génomique COVID-19 (RCanGéCO), qui n'a joué aucun rôle dans la rédaction de ce manuscrit.

Propriété intellectuelle du contenu : II s'agit d'un article en libre accès distribué conformément aux modalités de la licence Creative Commons Attribution (CC BY-NCND 4.0), qui permet l'utilisation, la diffusion et la reproduction de tout médium à la condition que la publication originale soit adéquatement citée, que l'utilisation se fasse à des fins non commerciales (c.-à-d., recherche ou éducation) et qu'aucune modification ni adaptation n'y soit apportée. Voir : https://creativecommons.org/licenses/by -nc-nd/4.0/deed.fr.

Partage des données : Santé publique Ontario (SPO) et le Bureau de santé du district d'Haliburton, Kawartha et Pine Ridge ne peuvent pas divulguer les données sous-jacentes pour ne pas compromettre la confidentialité des renseignements personnels, ce qui serait contraire à leurs obligations éthiques et juridiques. Un accès restreint aux données pourrait être possible en vertu de la Loi de 2004 sur la protection des renseignements personnels sur la santé, de la Loi sur l'accès à l'information et la protection de la vie privée, de l'Énoncé de politique des trois conseils : Éthique de la recherche avec des êtres humains et des politiques de SPO sur la protection des renseignements personnels et l'éthique. Les données sont accessibles aux chercheurs qui répondent aux critères de SPO pour la consultation de données confidentielles. La marche à suivre pour faire une demande d'accès à l'information auprès de SPO peut être consultée à cette adresse : https://www.publichealthontario.ca/fr/data -and-analysis/using-data/data-requests.

Remerciements : Les auteurs aimeraient souligner la contribution à l'analyse phylogénétique de Brenda Stanghini, Yao Chen et Aimin $\mathrm{Li}$, ainsi que du personnel du laboratoire de bio-informatique de Santé publique Ontario, et celle de Jared Simpson, de l'Ontario Institute for Cancer Research. Les auteurs remercient également Shelly Griffin, Mary Carr, Julie Parcells, les autres employés de l'ESLD et les employés du Bureau de santé du district d'Haliburton, Kawartha et Pine Ridge.

Accepté : Le 8 avril 2021

Correspondance : Michelle Murti, Michelle.murti@oahpp.ca 